\title{
Más allá del aprendizaje electrónico
}

\section{Beyond e-learning}

\section{Sr. Editor:}

Las tecnologías de información y comunicaciones (TICs) en educación han evolucionado progresivamente y se han convertido en herramientas útiles para facilitar la ganancia de conocimiento en los estudiantes. Tras revisar el artículo de Pérez-Martinot acerca de las TICs en la educación médica, hay conceptos relacionados al aprendizaje electrónico (e-learning) que no han sido abordados.

Las TICs en educación no solamente incluyen al e-learning. Si bien, los esfuerzos están dirigidos hacia el estudiante como principal beneficiario, también se articula con el e-teaching (enseñanza electrónica) y el e-assessment (evaluación electrónica) (1). Mientras que el e-learning se refiere a los recursos que el estudiante accede para aprender, el e-teaching se enfoca en aquellos instrumentos que facilitan al docente difundir contenidos. Como se menciona en el artículo, los docentes son "inmigrantes digitales" y es por esta limitación que se han creado aplicaciones que buscan atenuarla ya que no exigen altos conocimientos informáticos y ofrecen un entorno sencillo e intuitivo tales como las plataformas en línea para la administración de aulas virtuales y software para videoconferencias especialmente diseñado con opciones que permiten al docente interactuar con sus alumnos. Por ejemplo, en la Facultad de Medicina Alberto Hurtado (FMAH) se transmite en tiempo real las clases presenciales para los alumnos del internado. Aquellos internos que se encuentran realizando sus rotaciones en áreas rurales se conectan a la "sala" y "presencian" la clase, con la posibilidad de enviar sus preguntas al docente quien podrá resolverlas durante la misma.

Por otro lado, las tecnologías educativas dan soporte a las evaluaciones sumativas y formativas a través del e-assessment. Más aún, se hace indispensable su uso cuando se tratan de cursos totalmente impartidos en línea. Una de las herramientas más conocidas son los módulos para desarrollar cuestionarios en línea en los que se pueden crear diversos tipos de preguntas, como las de respuesta abierta y las de opción múltiple, y configurar alguna retroalimentación según las respuestas o puntajes obtenidos por el alumno. Otras soluciones útiles para el docente son la administración de bancos de preguntas y la gestión de calificaciones a través de un registro en línea. El e-assessment no sólo se aplica en las metodologías a distancia. También, puede ser llevado a cabo dentro del campus universitario. En la FMAH se hace uso del e-assessment en exámenes de conocimientos, evaluaciones entre pares (coevaluaciones) en el externado e internado y el llenado de rúbricas en las evaluaciones clínicas objetivas estandarizadas.

Mas adelante, en el artículo se menciona el uso limitado de las computadoras en el aula y su aún menor empleo por parte de los docentes. Pues, esta afirmación no indica necesariamente que el uso de tecnologías en las clases sea bajo. Al contrario, a partir de la masificación de los dispositivos móviles, como tablets y smartphones, los usuarios tienen mayor acceso a la información y ha impulsado la creación de software optimizado para este tipo de hardware. En efecto, el uso de esta tecnología en la educación se ha denominado mobile learning (m-learning) que por su complejidad no sólo se extiende al aprendizaje sino también a la enseñanza y a la práctica (2). El m-learning ha conseguido que el aprendizaje ya no sea exclusivo del salón de clases y que el estudiante tenga mayor autonomía. En educación médica, los estudiantes utilizan "apps" de literatura médica para esclarecer de forma rápida y oportuna las dudas que surgen mientras el docente dicta la clase. Inclusive, algunas aplicaciones dirigidas al campo hospitalario 
profesional pueden servir en el aula, como por ejemplo calculadoras clínicas y herramientas para el diagnóstico y tratamiento.

Tratar sobre TICs en educación médica podría resultar ser extenso, pero deben quedar aclarados sus principales conceptos ya que serán el punto de partida para que los docentes comprendan posteriormente sus efectos en el aprendizaje de sus estudiantes, los aspectos técnicos que implica, las estrategias para su buen uso y realizar propuestas innovadoras.

\section{Carlos Orellano ${ }^{1, a, b}$}

\section{Réplica del autor}

\section{Author reply}

\section{Sr. Editor:}

En relación a la carta titulada: "Más allá del aprendizaje electrónico", queremos agradecer al Dr. Orellano por compartir sus consideraciones con respecto al artículo y a su vez hacer las siguientes precisiones.

El e-learning es una metodología dentro del proceso de enseñanza-aprendizaje, que se lleva a cabo a través de Internet mediante la utilización de diferentes medios electrónicos. Comprende aspectos pedagógicos y tecnológicos. "El pedagógico, referido a la tecnología educativa como disciplina de las ciencias de la educación, vinculada a los medios tecnológicos y, la psicología educativa, con entornos curriculares, didácticos, evaluativos, interacción de medios, mediaciones y mediadores" (1).

El e-learning se encuentra en constante desarrollo y evolución, siendo la educación a distancia que se dirige en la actualidad llevada a cabo en plataformas inteligentes y personalizables por el mismo alumno (e-learning 3.0). Area y Adell (1)

\section{Correspondencia:}

Carlos Orellano

Correo electrónico: carlos.orellano@upch.pe

\section{REFERENCIAS BIBLIOGRÁFICAS}

1. Ellaway R, Masters K. AMEE Guide 32: e-Learning in medical education Part 1: Learning, teaching and assessment. Med Teach. 2008;30(5):455-73.

2. Masters K, Ellaway RH, Topps D, Archibald D, Hogue RJ. Mobile technologies in medical education: AMEE Guide No. 105. Med Teach. 2016;38(6):53749.

Recibido: 10/02/2018 mencionan: "Entre las características más destacadas de esta modalidad formativa están la desaparición de barreras espacio-temporales, la flexibilidad, la gestión real del conocimiento, el cambio de rol de alumno y profesor, la rapidez, el ahorro de costos, la actualización de contenidos, la comunicación constante, la autonomía del aprendizaje, etc. Se eliminan con ella los inconvenientes de la enseñanza tradicional, en la que el alumno se encontraba solo en su proceso de aprendizaje". En resumen, hablar de e-learning es referirse genéricamente a todos los procesos que involucran al de enseñanza-aprendizaje, es decir, el proceso de evaluación y autoevaluación, la participación de diferentes actores, principalmente el docente y el alumno, y el empleo para este fin de múltiples y variados dispositivos electrónicos, como el computador personal, los smartphones, tablets, entre otros.

Coincidimos en que no han sido abordados en su totalidad todos los temas relacionados al e-learning porque no sería posible dada su amplitud y constante evolución, además de no ser el objetivo principal de la revisión. De ser así, hubiéramos tenido que

Profesor asociado. Facultad de Medicina Alberto Hurtado, Universidad Peruana Cayetano Heredia. Lima, Perú.

Médico Oftalmólogo; ${ }^{b}$ Magister en Medicina y en Salud Pública con mención en Gerencia y Gobierno en Salud; 'Alumno del Programa de Doctorado en Medicina, Escuela de Postgrado Víctor Alzamora Castro, Universidad Peruana Cayetano Heredia. 
mencionar, además, los diferentes tipos de e-learning conocidos actualmente y que se pueden clasificar según el grado de presencialidad/no presencialidad en Direct e-Learning o e-Learning "presencial", Blended Learning o formación mixta, que incluye actividades tanto de la enseñanza tradicional como por Internet y Online learning o e-learning completamente en línea, donde no existe componente presencial alguno. Según los dispositivos utilizados, el e-learning se puede clasificar en: e-learning o aprendizaje "electrónico el cual se refiere a la formación online utilizando computadoras convencionales conectados a la red; el m-learning o aprendizaje con dispositivos móviles que es una modalidad de eLearning que se desarrolla en dispositivos móviles como PDAs, Tablet PCs y otros dispositivos con Windows $\mathrm{CE}$, teléfonos móviles, algunos dispositivos $\mathrm{mp} 3$ y mp4 de última generación e incluso consolas de juego portátiles con conexión a Internet y el $u$-Learning o aprendizaje ubicuo que vendría a ser una fusión de las dos modalidades anteriores y que permite acceder al aprendizaje independientemente del dispositivo empleado, pues el sistema adapta los contenidos, actividades y el modelo de interacción al dispositivo con el que se accede en cualquier momento y lugar (2).

Como dijimos, en el artículo de revisión se empleó el término e-learning en un sentido general, como alternativa general para el docente y el estudiante. No se describieron términos específicos como el e-teaching ni el e-assesment por no considerarse necesarios en dicha revisión; sin embargo, fueron tratados indirectamente en el texto. Se menciona la importancia del e-learning dentro de la educación médica, su evolución a través del tiempo (tabla 2) y los recursos que éste puede emplear (tabla 3) tanto en computadores personales como en dispositivos móviles. No se pretendió abarcar todas las diferentes ramificaciones que puede tener éste en materia de su utilidad para el docente específicamente ni en el campo de la evaluación del aprendizaje.

El rol del e-learning en la enseñanza de la medicina es cada día más importante. Cada vez son más las plataformas que permiten las videoconferencias y la interacción en tiempo real entre profesores y alumnos. Estas están siendo aplicadas en las diferentes escuelas de enseñanza de ciencias médicas en todo el mundo. La UPCH pionera en el Perú en investigación y educación médica no podía quedarse atrás en este aspecto. Sin embargo, no es suficiente. Es importante también, el desarrollo de aplicaciones interactivas, amigables para el usuario no experto, para complementar aquellos cursos de las diferentes especialidades médicas que por cuestiones de currículo no disponen del tiempo suficiente para ser expuesto a los alumnos. Es el caso del curso de oftalmología en el pregrado, por ejemplo, ha motivado en el autor del artículo de revisión, la necesidad de desarrollar un aplicativo web para la enseñanza de oftalmología básica dirigido a médicos no especialistas y estudiantes de medicina en general que próximamente será publicada.

Las evaluaciones son importantes y necesarias dentro de todo proceso de enseñanza-aprendizaje. Son de utilidad tanto para el docente como para el alumno. Para el primero, para evaluar la enseñanza impartida $\mathrm{y}$ hacer las modificaciones pertinentes y para el segundo, en la autoevaluación de los conocimientos adquiridos. Estas evaluaciones pueden ser a través de cuestionarios presenciales, en línea o e-assessment; sin embargo, su importancia o aplicación no fueron considerados, tampoco, como objetivo de la presente revisión.

El artículo hace referencia explícitamente al uso de computadores en el salón de clase. Es innegable que con el uso de dispositivos móviles (smartphones, tablets, etc), el usuario tiene mayor acceso a la información. El m-learning, la manera como se denomina actualmente a este tipo de aprendizaje a través de dispositivos móviles, es un tipo de e-learning que potencia sus beneficios y estamos seguros de su prometedora evolución. Una vez más, queremos resaltar que nuestros esfuerzos deben estar dirigidos al desarrollo de más "apps" nacionales que reflejen nuestra realidad.

Finalmente, el artículo de revisión intenta presentar al e-learning como una alternativa a la enseñanza de la medicina tradicional para que pueda ser implementada cada vez más dentro de la enseñanza médica tanto por docentes como por alumnos y motivarlos al desarrollo de materiales educativos propios. Definitivamente el artículo tiene limitaciones en cuanto a la cantidad de conceptos por definir, pero estamos seguros que servirá de punto de partida para futuras revisiones.

Consideramos que el mensaje de la carta al editor coincide con nuestra intención de motivar al lector a seguir profundizando en el tema y aplicar los nuevos conceptos que irá descubriendo.

Manuel Pérez Martinot ${ }^{1, a, b, c}$ 


\section{Correspondencia:}

manuel.perez.m@upch.pe

\section{REFERENCIAS BIBLIOGRÁFICAS}

1. Area M, Adell J. e-Learning: Enseñar y aprender en espacios virtuales. En J. De Pablos(Coord): Tecnología Educativa. La formación del profesorado en la era de Internet. Aljibe, Málaga; 2009.p. 391424. (Citado el 15 de mayo del 20189 Disponible en: http://tecedu.webs.ull.es/textos/eLearning.pdf

2. García-Peñalvo FJ, Pardo AMS. Una revisión actualizada del concepto de eLearning: Décimo Aniversario. Teoría la Educ Educ y Cult en la Soc la Inf. 2015;16(1):119-44. 